\title{
Limited inter-occasion variability in relation to inter-individual variability in chemotherapy-induced myelosuppression
}

\author{
Emma K. Hansson ${ }^{1}$, Johan Wallin ${ }^{1}$, Henrik Lindman ${ }^{2}$, Marie Sandström ${ }^{1,3}$, \\ Mats O. Karlsson ${ }^{1}$, Lena E. Friberg ${ }^{1}$ \\ ${ }^{1}$ Department of Pharmaceutical Biosciences, Uppsala University, Box 591, SE -751 24 \\ Uppsala, Sweden \\ ${ }^{2}$ Department of Oncology, Radiology and Clinical Immunology, Uppsala University \\ Hospital, 75185 Uppsala, Sweden \\ ${ }^{3}$ AstraZeneca ReD, SE-151 85, Södertälje, Sweden
}

\author{
Corresponding author: \\ Emma Hansson \\ Phone: + 46184734303 \\ Fax: $\quad+46184714003$ \\ Email: emma.hansson@farmbio.uu.se
}

Financial support: This work was supported by the Swedish Cancer Society, Sweden. Lena Friberg was supported by Knut and Alice Wallenberg foundation, Sweden. Johan Wallin was supported by the Swedish Academy of Pharmaceutical Sciences.

\begin{abstract}
Purpose: A previously developed semi-physiological model of chemotherapy-induced myelosuppression has shown consistent system-related parameter and inter-individual variability (IIV) estimates across drugs. A requirement for dose individualization to be useful is relatively low variability between treatment courses (IOV) in relation to IIV. The objective of this study was to evaluate and compare magnitudes of IOV and IIV in myelosuppression model parameters across six different anti-cancer drug treatments.

Methods: Neutrophil counts from several treatment courses following therapy with docetaxel, paclitaxel, epirubicin-docetaxel, 5-fluorouracil-epirubicin-cyclophosphamide, topotecan and etoposide were included in the analysis. The myelosuppression model was fitted to the data using NONMEM VI. IOV in the model parameters baseline neutrophil counts $\left(\mathrm{ANC}_{0}\right)$, mean transit time through the non-mitotic maturation chain (MTT) and the parameter describing the concentration-effect relationship (Slope) were evaluated for statistical significance $(\mathrm{P}<0.001)$.

Results: IOV in MTT was significant for all the investigated datasets, except for topotecan, and was of similar magnitude (8-16 CV \%). IOV in Slope was significant for docetaxel, topotecan and etoposide (19-39 CV \%). For all six investigated datasets the IOV in myelosuppression parameters was lower than the IIV. There was no indication of systematic shifts in the system- or drug sensitivity-related parameters over time across data sets.

Conclusion: This study indicates that the semi-physiological model of chemotherapyinduced myelosuppression has potential to be used for prediction of the time-course of myelosuppression in future courses and is thereby a valuable step towards individually tailored anticancer drug therapy.
\end{abstract}

Keywords: Hematologic toxicity, pharmacodynamics, NONMEM, inter-occasion variability, anti-cancer drugs 


\section{Introduction}

Traditionally the initial dose level of most chemotherapeutic agents is based on body surface area (BSA) $\left(\mathrm{mg} / \mathrm{m}^{2}\right)$. In spite of this attempt for dose individualization toxicity and efficacy vary considerable among patients [1] where myelosuppression is the most common and often dose-limiting adverse event [2]. For patients with unacceptable toxicity the next dose is generally reduced in more or less crude predefined steps and/or the treatment interval is prolonged, whereas when little or no toxicity is observed, dose escalations are seldom performed outside clinical trials. Consequently patients may experience suboptimal tumor effects since a low dose intensity and/or lack of hematological toxicity is associated with shorter survival [3-6].

In an optimal dosing strategy the desired antitumoral effects have to be carefully balanced against the side effects for each individual. A way to do this could be to use the observed neutrophil counts from one treatment cycle as a base for dose adjustment in the next cycle. A model-based tool for efficient dose individualization based on neutrophil counts has recently been developed [7]. This tool uses a maximum a posteriori (Bayesian) approach to calculate a suitable dose for the next course based on a previously developed population pharmacokinetic-pharmacodynamic model for chemotherapy-induced myelosuppression [8] and observed neutrophil counts. The value of the dose-individualization tool depends on relatively low variability between treatment courses, inter occasion variability (IOV), in myelosuppression model parameters in relation to the interindividual variability (IIV), i.e. to which extent the observed neutrophil counts are predictable at the next course within the same patient.

A semi-physiological model that de- scribes the magnitude and duration of myelosuppression following anticancer treatment has previously been developed [8]. The model (Fig. 1) is composed of five compartments which imitate the myelopoiesis. One compartment represents proliferating cells in the bone marrow and is linked via three transit compartments, mimicking cell maturation, to a compartment corresponding to circulating observed neutrophils. Included is also a feedback mechanism increasing the neutrophil production when the number of circulating neutrophils in the blood are reduced representing e.g. the action of endogenous granulocyte colony stimulating factor (G-CSF). The drug is assumed to act by inhibiting the proliferation rate and inducing cell loss. In most cases it is sufficient to use a single parameter related to the drug concentration-effect relationship, i.e. a linear drug effect parameter (Slope). The estimated parameters associated to the hematopoietic system are the baseline neutrophil count $\left(\mathrm{ANC}_{0}\right)$, the mean transit time through the maturation chain (MTT) and the feedback factor gamma $(\gamma)$.

The semi-physiological myelosuppression model has been applied to several different anticancer drugs and found applications in many areas of drug development [9]. Consistency in the system-related parameter estimates and in the magnitude of IIV in the parameters across drugs have been reported [8]. However, there is limited information on the within individual variability between courses (IOV) in the estimated parameters. The aim of the present study was to evaluate IOV in myelosuppression model parameters and compare their magnitudes with IIV estimates across six different treatments to assess the semiphysiological model's potential as a tool for individual dose adjustments based on observed neutrophil counts. 




Fig. 1 The semi-physiological model of myelosuppression with the system related model parameters $\left(\mathrm{ANC}_{0}\right.$ ), mean transit time (MTT), feedback factor $\gamma$ and the drug-effect parameter (Slope). $\mathrm{K}_{t r}$, proliferation rate constant; $\mathrm{K}_{\text {circ }}$, elimination rate constant for circulating neutrophils; $\left(\mathrm{ANC}_{0} / \mathrm{ANC}\right)^{\gamma}$ feedback loop from the circulating neutrophils

\section{Patients and Methods}

\section{Patients and treatment}

Neutrophil counts from several treatment courses were available following therapy with docetaxel, paclitaxel, epirubicin-docetaxel, 5-fluorouracil-epirubicin-cyclophosphamide, topotecan and etoposide. Data from treatment cycles where patients were known to have received granulocyte colony stimulating factor (G-CSF) therapy were excluded from the analysis. All patients signed informed consent forms and the studies were in accordance with the Declaration of Helsinki and approved by local ethics committees. A summary of the analyzed datasets, number of patients, number of treatment cycles per patients, number of available neutrophil observations and number of neutrophil observations per patient and treatment cycle is presented in Table 1.

\section{Docetaxel}

Neutrophil counts from 244 metastatic breast cancer patients treated with docetaxel were included in the analysis [10]. The patients were part of the active control group in a clinical trial studying the combination treatment of capecitabine and docetaxel. Initial dose level was 100 $\mathrm{mg} / \mathrm{m}^{2}$ of docetaxel administered as a 1-hour intravenous infusion in a 3-week cycle. Dose reductions were based on hematological and non-hematological toxicity and resulted in a final dose range of $50-100 \mathrm{mg} / \mathrm{m}^{2}$.

Table 1. Data summary of the analyzed data sets

\begin{tabular}{l|cccc}
\hline Data set & n patients & $\begin{array}{c}\text { n cycles/ patient } \\
\text { median (range) }\end{array}$ & $\begin{array}{c}\text { n neutrophil } \\
\text { observations }\end{array}$ & $\begin{array}{c}\text { n neutrophil observations } \\
\text { /patient \& cycle } \\
\text { median (range) }\end{array}$ \\
\hline $\begin{array}{l}\text { Docetaxel } \\
\text { Paclitaxel }\end{array}$ & 244 & $4(1-16)$ & 2262 & $1.6(1.1-3.2)$ \\
$\begin{array}{l}\text { Epirubicin-docetaxel } \\
\text { 5-Fluorouracil- } \\
\text { epirubicin- } \\
\text { cyclophosphamide }\end{array}$ & 45 & $3(1-11)$ & 523 & $2.6(0.9-3.5)$ \\
$\begin{array}{l}\text { Topotecan } \\
\text { Etoposide }\end{array}$ & 41 & $4(1-9)$ & 659 & $3.6(2.9-4.7)$ \\
\hline
\end{tabular}

${ }^{a}$ Data from 11 out of 18 treatment cycles were analyzed as only one individual contributed > 11 cycles. 


\section{Paclitaxel}

The paclitaxel data included neutrophil counts from 45 patients with different cancer forms [11]. Paclitaxel was administered as a 3-hour infusion with an initial dose of $175 \mathrm{mg} / \mathrm{m}^{2}$ every $3^{\text {rd }}$ week. Doses were adjusted based on hematological and non-hematological toxicity resulting in a final dose range of 110-232 $\mathrm{mg} / \mathrm{m}^{2}$.

\section{Epirubicin-docetaxel}

The epirubicin-docetaxel (ET) dataset included 41 advanced breast cancer patients [12]. Epirubicin was given in a 3 -week cycle as a 1-hour infusion followed by a 1-hour free interval and then a 1-hour infusion of docetaxel. Initial doses were $75 / 70 \mathrm{mg} / \mathrm{m}^{2}$ with escalated/ reduced doses in the following cycles based on leukocyte and platelet counts according to the study protocol.

\section{5-Fluorouracil - epirubicin - cyclophosphamide}

Sixty breast cancer patients treated with either standard or tailored 5-fluorouracil-epirubicin-cyclophosphamide (FEC) regimen were included in the analysis [13]. The treatment was administered every $3^{\text {rd }}$ week as a 15 minute infusion of cyclophoshamide followed by 5 - fluorouracil given as an intravenous bolus dose and epirubicin given either as a bolus or as a 1-hour infusion. The initial doses of 5-fluorouracil, epirubicin and cyclophosphamide were in the first treatment cycle for standard FEC 600/60/600 mg/ $\mathrm{m}^{2}$, respectively, and for the tailored therapy $600 / 75 / 900 \mathrm{mg} / \mathrm{m}^{2}$, respectively. Subsequent doses were reduced based on toxicity in the standard therapy and in the tailored therapy doses were stepwise escalated or decreased based on the observed nadir and the dosing day leukocyte/platelet count according to a dose escalation/reduction protocol.

\section{Topotecan}

Data from 26 patients with various types of solid tumors treated with topotecan as single anticancer drug therapy were included in the analysis [14]. Initial dose level was $6 \mathrm{mg} / \mathrm{m}^{2}$ administered as a 24hour intravenous infusion every $3^{\text {rd }}$ week. No dose adjustments were performed according to the study protocol.

\section{Etoposide}

Data from 44 patients with solid tumors and hematological malignancies who received two treatment courses of a 3 -day continuous infusion of etoposide in a 28 day cycle were analyzed $[15,16]$. The patients were randomized to either standard dosing with a total dose of 375 $\mathrm{mg} / \mathrm{m}^{2}$ or concentration guided dosing where the total delivered dose ranged from $225-789 \mathrm{mg} / \mathrm{m}^{2}$ following dose adjustments

\section{Data analysis}

To describe the pharmacokinetics (PK) and pharmacodynamics (PD) following single-agent or combined chemotherapy non-linear mixed effects modeling was applied using the first order conditional estimation (FOCE) method in NONMEM version VI [17]. This approach estimates the typical (mean) value of parameters and can provide separate estimates of inter-individual (IIV), interoccasion (IOV) and residual error variability.

The model building process was guided by graphical diagnostics within the R-based software Xpose version 4.0 [18] (http://xpose.sourgeforge.net) and the change in objective function value (OFV) computed by NONMEM in the likelihood ratio test. For two nested models the differences in OFV is equal 
to minus twice the log likelihood and approximately $\chi^{2}$ distributed. A difference in OFV of $>10.83$ corresponds to a significance level of $\mathrm{P}<0.001$ for one additional parameter.

\section{Pharmacokinetics}

For the docetaxel data set no individual PK data were available and typical population PK parameters were used to describe the concentration-time profiles of the drug [19]. The PK of paclitaxel (average 3.5 PK samples per patient from treatment course 1 and 3) was described using individual PK parameters from a previously determined PK model for the data set [11]. On average 4.5 PK samples per patient at 18 occasions from $16 \mathrm{pa}$ tients were used to describe the PK of ET using individual PK parameters from a previous PK model for the ET dataset (12). For the FEC dataset concentration time- profiles were obtained using doses and individual $\mathrm{PK}$ parameters $(22 \%$ of the patients, 2-7 samples per patient ) or typical population parameters when no PK information was available (78\% of the patients) from a previously developed PK model [13].

The individual concentration-timecourse of topotecan and etoposide were derived from observed plasma concentrations and PK models developed by Legér et al. [20] and Toffoli et al. [21], respectively. For etoposide two plasma concentration samples per patient and treatment course were sampled [14] and for topotecan 185 plasma concentration measurements of total topotecan were obtained in the first treatment course $[15,16]$.

When pharmacokinetic observations were lacking and population typical values were used in describing the PK of the drugs, all IIV were assumed to be in myelosuppression and will likely result in an inflated IIV in the Slope parameter.

\section{Pharmacodynamic modeling of myelosuppression}

The semi-physiological model of myelosuppression was fitted to the neutrophil data. The model structure was the same as in the original publication [8] except that the half-life of circulating neutrophils was fixed to the literature value of 7 hours [22] and the neutrophil data were Box-Cox transformed ANC $_{\text {transformed }}=$ $\left.\left(\mathrm{ANC}^{\lambda}-1\right) / \lambda\right)$ with $\lambda=0.2$ prior to the analysis as this transformation resulted in residuals with a symmetrical distribution around zero $[23,24]$.

The subroutine PRIOR within NONMEM [17, 25] was used to be able to estimate separate drug effect parameters (Slope) for the co-administered drugs in the ET and FEC regimens. The prior information was incorporated as a frequentist prior where a penalty is added to the objective function on deviation from the prior. The estimated Slope parameter for docetaxel (typical value and standard error) in the single drug data set was used as informative prior for the docetaxel drug effect parameter when analyzing the ET data set. The obtained population estimate and standard error of the epirubicin Slope parameter in the ET regimen was thereafter used as prior when modeling FEC. The drug effects were assumed to be additive as this assumption has previously been shown to be reasonable for leukocytes [12].

The random IIV and IOV were modeled in terms of eta $(\eta)$ and kappa $(x)$ variables, respectively [26]. The $\eta s$ and us were assumed to be log-normally distributed parameters both with mean zero and variances $\omega^{2}$ and $\pi^{2}$, respectively. The IOV and IIV variance parameters were constant across all occasions. The random residual error, the differences between the observed neutrophil count and the model predicted neutrophil count, was modeled as an additive com- 
ponent (on Box-Cox scale).

As in the original publication of the semi-physiological model of myelosuppression [8] IIV was included for the model parameters $\mathrm{ANC}_{0}$, MTT and Slope for all datasets. IOV was evaluated for statistical significance $(\mathrm{P}<0.001)$ using OFV in the likelihood ratio test for $\mathrm{ANC}_{0}$, MTT and Slope. One occasion was defined as one treatment course with the nominal cycle length of 21 or 28 (etoposide) days. To exclude the possibility of time-dependent and non-random variability between occasion's linear changes with time in $\mathrm{ANC}_{0}$, MTT and Slope were estimated and evaluated for statistical significance $(\mathrm{P}<0.001)$. Timedependent changes in the model fit were also evaluated by graphical assessment of the conditional weighted residuals (CWRES).

Reliability in the parameter estimates were determined by standard errors obtained from the $S$ matrix ( $\mathrm{R}$ matrix for topotecan) in NONMEM due to long run times and as these standard errors are good approximations to the standard errors obtained by the in NONMEM default sandwich matrix and to a nonparametric bootstrap procedure [27].

The magnitude of IOV in the myelosuppression model parameters in relation to the IIV was explored by comparison of the variability in simulated nadir counts. The final parameter estimates for each of the six analyzed data sets were used to simulate 1000 time-courses of myelosuppression for all treatment regimes including only IIV, only IOV, or both IOV and IIV. The nadirs for the 1000 time-courses were identified and the distributions of simulated nadir counts, including only IIV, only IOV, or both IOV and IIV, were compared.

\section{Results}

The myelosuppression model could well characterize the neutrophil-time course following both the single-agent and combination therapy for all the investigated datasets and resulted in similar systemrelated parameter estimates as previously observed for other data sets $[8,28]$. For 5-fluorouracil, Slope was not significantly different from zero i.e. the drug effect for 5-fluorouracil could not be separated from the drug effect of epirubicin and cyclophosphamide with the present data (Table 2).

Estimated IIV and IOV in the model parameters and the decrease in residual errors after IOV inclusion are reported in Table 2. In accordance with previous results $[8,28]$ IIV in the $\mathrm{ANC}_{0}$ and Slope parameters were larger than IIV in MTT. IIV in ANC was similar across drugs (slightly higher for etoposide) while the IIV in Slope varied (22-62 \% $\mathrm{CV})$ between the different treatments. The IIV in Slope was lower in the drug combination data sets (where a common IIV parameter for Slope was estimated for the component drugs) compared to the single agent data. IIV in Slope for topotecan was estimated to be relatively high compared with the other investigated datasets.

IOV in MTT was significant and of similar magnitude (7.5-16 \% CV) for all the investigated datasets, except for topotecan where only IOV in Slope was significant to include. IOV in Slope was also found to be significant for docetaxel and etoposide. By inclusion of IOV in the myelosuppression model parameters the residual errors decreased on average $21 \%$ for all data sets with the highest decrease in residual errors observed for the paclitaxel and etoposide datasets (Table 2).

There were no significant time-dependent changes in parameters where 
Table 2. Typical population parameter estimates (relative SE \%) for final models including IOV. $\Delta$ residual error is the relative change in residual error after inclusion of IOV.

\begin{tabular}{|c|c|c|c|c|c|c|c|}
\hline Data set & $\begin{array}{c}\mathrm{ANC}_{0} \\
(\mathrm{x} \mathrm{10} / \mathrm{L}) \\
\end{array}$ & $\begin{array}{c}\begin{array}{c}\text { MTT } \\
\text { (hours) }\end{array} \\
\end{array}$ & $\begin{array}{l}\text { Slope 1 } \\
\left(\mu \mathrm{M}^{-1}\right)\end{array}$ & $\begin{array}{l}\begin{array}{l}\text { Slope 2 } \\
\left(\mu M^{-1}\right)\end{array} \\
\end{array}$ & $\gamma$ & $\begin{array}{c}\text { Residual } \\
\text { Error }^{\mathrm{a}}\end{array}$ & \\
\hline Docetaxel & $4.81(2.6)$ & $94.0(1.6)$ & $17.3(3.4)$ & - & $0.170(1.8)$ & $0.528(1.1)$ & \\
\hline Paclitaxel & $5.61(9.4)$ & $154(4.4)$ & $69.6(8.1)$ & - & $0.270(5.9)$ & $0.431(2.6)$ & \\
\hline $\begin{array}{l}\text { Epirubicin- } \\
\text { Docetaxel } \\
\text { e5-Fluorouracil- }\end{array}$ & $3.49(11)$ & $117(3.1)$ & ${ }^{\mathrm{b}} 17.8(32)$ & ${ }^{\mathrm{c}} 17.4(29)$ & $0.207(5.1)$ & $0.499(3.4)$ & \\
\hline $\begin{array}{l}\text { epirubicin- } \\
\text { cyclophosphamide }\end{array}$ & $4.56(5.3)$ & $184(3.2)$ & ${ }^{b} 32.2(47)$ & ${ }^{\mathrm{d}} 26.6(22)$ & $0.241(2.4)$ & $0.535(1.9)$ & \\
\hline Topotecan & $7.11(9.6)$ & $157(5.6)$ & $0.0370(27)$ & - & $0.275(8.6)$ & $0.472(1.1)$ & \\
\hline \multirow[t]{2}{*}{ Etoposide } & $5.69(9.8)$ & $162(6.9)$ & $0.128(11)$ & - & $0.170(3.4)$ & $0.492(4.5)$ & \\
\hline & $\begin{array}{c}\text { IIV ANC } \text { AN }_{0} \\
(\mathrm{CV} \%)\end{array}$ & $\begin{array}{c}\text { IIV MTT } \\
\text { (CV \%) }\end{array}$ & $\begin{array}{c}\text { IIV Slope } \\
\text { (CV \%) }\end{array}$ & $\begin{array}{c}\text { IOV ANC }_{0} \\
(\mathrm{CV} \%)\end{array}$ & $\begin{array}{c}\text { IOV MTT } \\
\text { (CV \%) }\end{array}$ & $\begin{array}{c}\text { IOV Slope } \\
\text { (CV \%) }\end{array}$ & $\begin{array}{c}\Delta \text { Residual } \\
\text { Error \% }\end{array}$ \\
\hline Docetaxel & $33(5.9)$ & $9.0(19)$ & $37(7.0)$ & - & $16(4.8)$ & $19(12)$ & -17 \\
\hline Paclitaxel & $36(13)$ & $17(22)$ & $39(20)$ & - & $16(8.5)$ & - & -41 \\
\hline $\begin{array}{l}\text { Epirubicin- } \\
\text { Docetaxel }\end{array}$ & $37(15)$ & $12(21)$ & $f_{22}(23)$ & - & $8.0(20)$ & - & -17 \\
\hline $\begin{array}{l}\text { 5-Fluorouracil- } \\
\text { epirubicin- } \\
\text { cyclophosphamide }\end{array}$ & $28(15)$ & $16(13)$ & $f_{23}(14)$ & - & $7.5(11)$ & - & -7.0 \\
\hline Topotecan & $32(27)$ & $15(34)$ & $62(45)$ & - & - & $28(39)$ & -3.3 \\
\hline Etoposide & $47(15)$ & $23(24)$ & $28(42)$ & - & $12(39)$ & $39(24)$ & -38 \\
\hline
\end{tabular}

${ }^{a}$ On Box-Cox transformed scale

${ }^{\mathrm{b}}$ Epirubicin

'Docetaxel

${ }^{\mathrm{d} C y c l o p h o s p h a m i d e}$

'Slope for 5-fluorouracil not significantly different from zero.

${ }^{\mathrm{f}}$ common IIV parameter for Slope for the component drugs

IOV was included indicating that the estimated $x$ s were random and not time dependent. Significant linear trends over time were however found in $\mathrm{ANC}_{0}$ for the FEC and etoposide datasets for which $\mathrm{IOV}$ were not significant in $\mathrm{ANC}_{0}$. The estimated trend over time corresponds to a decrease in $\mathrm{ANC}_{0}$ from 4.56 to 3.81 x $10 \%$ L neutrophils 15 weeks after first treatment for the typical patient treated with FEC. For etoposide an increase in $\mathrm{ANC}_{0}$ from 5.69 to $6.32 \times 10^{9} / \mathrm{L}$ neutrophils was estimated 4 weeks after first treatment. No time-dependent changes in the model fit (Figure 2) were visible in the graphical assessment of CWRES.

In all six data sets, the contribution to the variability in neutrophil nadir was clearly lower from IOV than from IIV as shown in Figure 3. The impact of the estimated IIV and IOV on the timecourses of myelosuppression is visualized in Figure 4 for 20 simulated individuals.

\section{Discussion}

The time-course of neutrophils following chemotherapy is here described for six different anti-cancer drug treatments for which the estimated parameters are reported. The semi-physiological myelosuppression model has not previously been applied for neutrophils for the here used data sets on docetaxel, ET, FEC and topotecan, and for none of the data sets has IOV previously been characterized. For all six investigated dataset the impact of IOV on the variability in nadir counts was lower in relation to the IIV.

Typically IIV parameters were of similar magnitudes across drugs but the estimated IIV in Slope for topotecan was high $(62 \%)$ which may be explained by a heterogeneous patient population with advanced disease. The estimate of the system-related parameter $\mathrm{ANC}_{0}$ for topotecan $\left(7.1 \times 10^{9} / \mathrm{L}\right)$ was also higher 


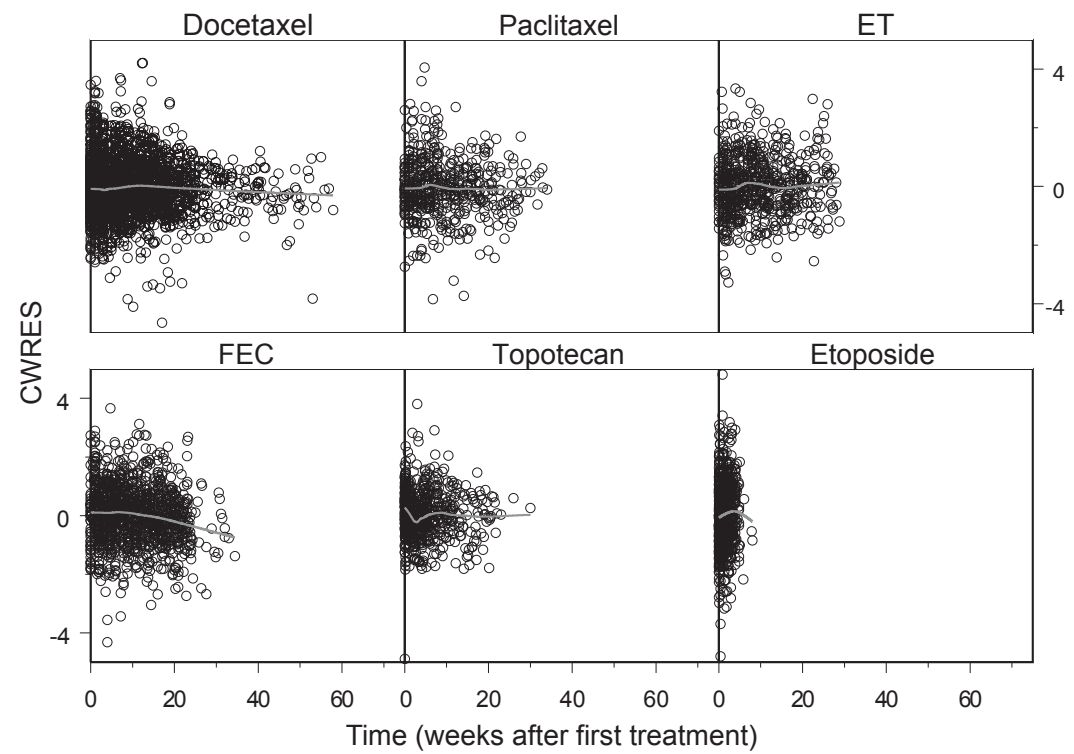

Fig. 2 Graphical evaluation of time-dependent changes in the model fit by conditional weighted residuals (CWRES) versus time for the six investigated datasets.

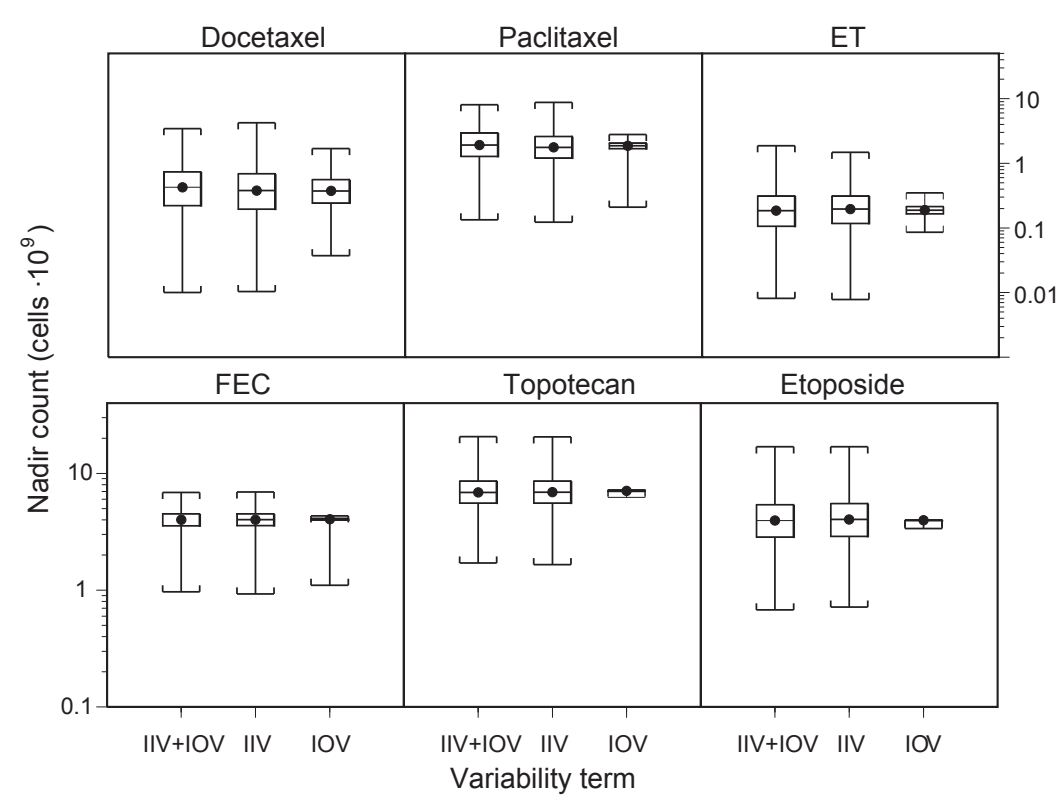

Fig. 3 Box-plots of simulated nadir distributions for all six treatment regimens including both IOV and IIV, only IIV or only IOV. The solid circle corresponds to the median, the top and bottom of the box the $25^{\text {th }}$ and $75^{\text {th }}$ percentiles and the whiskers to the maximum and minimum of the simulated nadir counts 


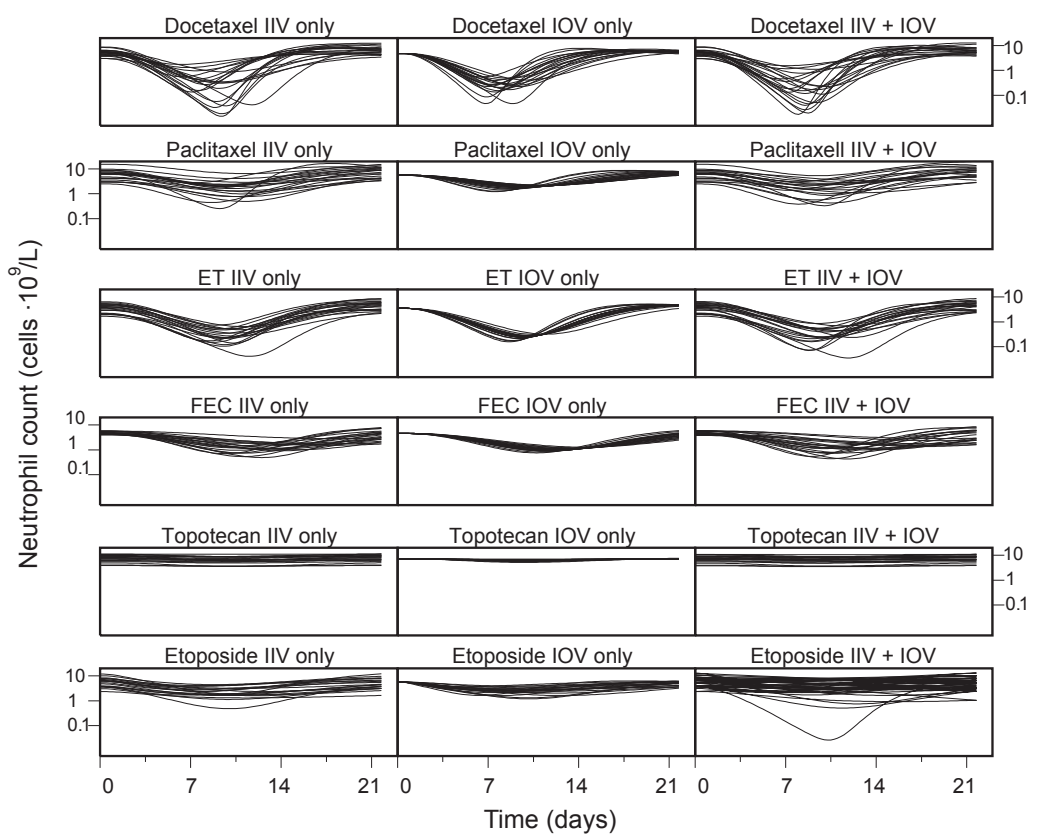

Fig. 4 Twenty simulated individual time-courses of myelosuppression including IIV only, IOV only or IIV and IOV for all the six investigated datasets

than for the other investigated dataset in the current and previous studies [8] but was in accordance with the observed initial baseline neutrophil count, $6.8 \mathrm{x}$ $10^{9} / \mathrm{L}$.

IOV in MTT was significant for all the investigated datasets except for topotecan. This may indicate that MTT is a parameter which influences most of the neutrophil observations and therefore inclusion of IOV in MTT results in a significant improvement of the fit. Potential variability between treatment courses in drug sensitivity and baseline neutrophil count within an individual appeared however of lower importance.

For none of the data sets did the drug sensitivity of the bone marrow increase with time and typically potential changes in pre-treatment neutrophil counts over time were predicted by the model. Significant linear changes with time in $\mathrm{ANC}_{0}$ were however found for FEC and etoposide, but the observed trends were of small magnitudes and in opposite directions. A decrease in $\mathrm{ANC}_{0}$ over time was observed for FEC in contrast to an increase over time for etoposide. As no time-dependent trends were observed in any of the other investigated datasets it is hard to draw any conclusion from the findings.

IOV in myelosuppression model parameters for oral and intravenous administered topotecan as mono therapy or in combination with cisplatin have been reported previously by Léger et al [29]. The estimated IOV in Slope and MTT were $93 \%$ and $22 \%$, respectively. A part of the large IOV in Slope was speculated to be caused by the oral administration route and the different treatment sequences of topotecan and cisplatin between cycle 1 and 2 . In our analysis only IOV in Slope (29 CV \%) was found significant for topotecan whereas IOV in 
MTT was not supported by the data. In the Léger study the first order estimation method was used and the estimated variability parameters were associated with large confidence intervals and thus there may not be a conflict between their findings and ours. For both studies on topotecan, the estimated IOV in relation to the IIV was lower.

PK were not determined in all treatment cycles in any of the analyzed datasets and therefore potential IOV in PK was likely incorporated in residual error estimates or in IOV of the myelosuppression model parameters. IOV in pharmacokinetic parameters for the component drugs of the ET, FEC and topotecan regimens has earlier been shown to be limited and less than the IIV [12, 13, 29]. The estimated IOV in clearance ranged between 14-18 \%.

Two alternative a posteriori dosing strategies to traditional dose adjustments in predefined steps are pharmacokinetic and pharmacodynamic adaptive control [30]. The adaptive control strategies have been successfully evaluated in the clinic for some antineoplastic agents [31, 32]. However, except for methotrexate, these dose-adaptation methods have not found widespread use with the primary reason being the poorly defined relationship between plasma drug concentrations, therapeutic effect and/or toxicity. Neither has the suggested dosing strategies (except for methotrexate) yet prospectively proved benefit in terms of increased response and reduced toxicity $[31,32]$. By using the semi-physiological myelosuppression model [8] as a tool for dose individualization based on observed neutrophil counts both individual pharmacokinetic and pharmacodynamic differences between patients may be accounted for and doses can be tailored to acceptable neutropenia.

In conclusion, for all six investigated datasets of chemotherapy-induced my- elosuppression, the estimated impact of IOV in myelosuppression parameters on the variability in nadir counts was clearly lower than the IIV. No indication of systematic shifts in the systemor drug sensitivity-related parameters over time across data sets was present. The time-course of myelosuppression is thereby shown to be predictable within a patient which supports the use of the recently developed model-based dose individualization tool based on observed neutrophil counts [7]. This study is thus a valuable step towards individually tailored anticancer drug therapy when myelosuppression is dose-limiting.

\section{Acknowledgment}

The authors would like to thank F. Hoffman La-Roche ltd. (docetaxel), Peter Nygren, MD (paclitaxel) and Mark Ratain, MD (etoposide) for kindly providing the data.

\section{References}

1. Sawyer M, Ratain MJ. Body surface area as a determinant of pharmacokinetics and drug dosing. Investigational New Drugs. 2001;19:171-177.

2. Crawford J, Dale DC, Lyman GH. Chemotherapy-induced neutropenia Risks, consequences, and new directions for its management. Cancer. 2004 Jan;100:228-237.

3. Mayers C, Panzarella T, Tannock IF. Analysis of the prognostic effects of inclusion in a clinical trial and of myelosuppression on survival after adjuvant chemotherapy for breast carcinoma. Cancer. 2001;91:2246-2257.

4. Saarto T, Blomqvist C, Rissanen P, Auvinen A, Elomaa I. Haematological toxicity: A marker of adjuvant chemotherapy efficacy in stage II and III breast cancer. British Journal of Cancer. 1997;75:301-305. 
5. Cameron DA, Massie C, Kerr G, Leonard RCF. Moderate neutropenia with adjuvant CMF confers improved survival in early breast cancer. British Journal of Cancer. 2003 Nov;89:18371842.

6. Poikonen P, Saarto T, Lundin J, Joensuu $\mathrm{H}$, Blomqvist C. Leucocyte nadir as a marker for chemotherapy efficacy in node-positive breast cancer treated with adjuvant CMF. British Journal of Cancer. 1999;80:1763-1766.

7. Wallin J, Friberg LE, Karlsson MO. A tool for neutrophil guided dose adaptation in chemotherapy. Comp Meth Prog Biomed. 2009;93:283-291.

8. Friberg LE, Henningsson A, Maas H, Nguyen L, Karlsson MO.

Model of chemotherapy-induced myelosuppression with parameter consistency across drugs. J Clin Oncol. 2002 Dec;20:4713-4721.

9. Karlsson MO, Anehall T, Friberg LE, et al. Pharmacokinetic/ pharmacodynamic modelling in oncological drug development. Basic \& Clinical Pharmacology \& Toxicology. 2005;96:206-211.

10. O’Shaughnessy J, Miles D, Vukelja S, et al. Superior survival with capecitabine plus docetaxel combination therapy in anthracycline-pretreated patients with advanced breast cancer: Phase III trial results. J Clin Oncol. 2002 Jun;20:2812-2823.

11. Henningsson A, Sparreboom A, Sandstrom M, et al. Population pharmacokinetic modelling of unbound and total plasma concentrations of paclitaxel in cancer patients. European Journal of Cancer. 2003;39:1105-1114.

12. Sandstrom M, Lindman H, Nygren P, Lidbrink E, Bergh J, Karlsson MO. Model describing the relationship between pharmacokinetics and hematologic toxicity of the epirubicindocetaxel regimen in breast cancer patients. J Clin Oncol. 2005 Jan 20;23:413-421.
13. Sandstrom M, Lindman $H$, Nygren P, Johansson M, Bergh J, Karlsson MO. Population analysis of the pharmacokinetics and the haematological toxicity of the fluorouracil-epirubicincyclophosphamide regimen in breast cancer patients. Cancer Chemother Pharmacol. 2006 Aug;58:143-156.

14. Vanwarmerdam LJC, Huinink WWB, Rodenhuis S, et al. Phase-I Clinical and Pharmacokinetic Study of Topotecan Administered by a 24-Hour Continuous-Infusion. J Clin Oncol. 1995 Jul;13:1768-1776.

15. Ratain MJ, Mick R, Schilsky RL, Vogelzang NJ, Berezin F. Pharmacologically Based Dosing of Etoposide - a Means of Safely Increasing Dose Intensity. J Clin Oncol. 1991 Aug;9:1480-1486.

16. Ratain MJ, Schilsky RL, Choi KE, et al. Adaptive-Control of Etoposide Administration - Impact of Interpatient Pharmacodynamic Variability. Clinical Pharmacology \& Therapeutics. 1989;45:226-233.

17. Beal S, Sheiner L. NONMEM Users Guides. NONMEM Project Group. NONMEM Project Group, University of California at San Francisco ed. San Francisco; 2006.

18. Jonsson EN, Karlsson MO. Xpose:an Splus based population Pharmacokinetic/pharmacodynamic model bulding aid for NONMEM. Comput Methods Programs Biomed 1999;58:51-64.

19. Bruno R, Vivier N, Vergniol JC, DePhillips SL, Montay G, Sheiner LB. A population pharmacokinetic model for docetaxel (Taxotere(R)): Model building and validation. Journal of Pharmacokinetics and Biopharmaceutics. 1996;24:153-172.

20. Leger F, Loos WJ, Fourcade J, et al. Factors affecting pharmacokinetic variability of oral topotecan: a population analysis. British Journal of Cancer. 2004;90:343-347.

21. Toffoli G, Corona G, Sorio R, et al. Population pharmacokinetics and pharmacodynamics of oral etoposide. British Journal of Clinical Pharmacology. 2001;52:511-519. 
22. Cartwright GE, Athens JW, Wintrobe MM. The Kinetics of Granulopoiesis in Normal Man. Blood. 1964;24:780-803.

23. Karlsson MO, Port RE, Ratain MJ, Sheiner LB. A Population-Model for the Leukopenic Effect of Etoposide. Clinical Pharmacology \& Therapeutics. 1994;55:152-152.

24. Friberg LE, Brindley CJ, Karlsson MO, Devlin AJ. Models of schedule dependent haematological toxicity of $2^{\prime}$-deoxy-2 '-methylidenecytidine (DMDC). European Journal of Clinical Pharmacology. 2000;56:567-574.

25. Gisleskog PO, Karlsson MO, Beal SL. Use of prior information to stabilize a population data analysis. J Pharmacokinet Pharmacodyn. 2002 Dec;29:473-505.

26. Karlsson MO, Sheiner LB.

The Importance of Modeling Interoccasion Variability in Population Pharmacokinetic Analyses. Journal of Pharmacokinetics and Biopharmaceutics. 1993;21:735-750.

27. Gibiansky L. Precision of Parameter Estimates: Covariance Step (\$COV) versus Bootstrap Procedure. PAGE 16 (2007) Abstr 1106 [wwwpagemeetingorg/?abstract=1106]. 2007.

28. Kloft C, Wallin J, Henningsson A, Chatelut E, Karlsson MO. Population pharmacokinetic-pharmacodynamic model for neutropenia with patient subgroup identification: Comparison across anticancer drugs. Clinical Cancer Research. 2006 Sep;12:5481-5490.

29. Leger F, Loos WJ, Bugat R, et al. Mechanism-based models for topotecaninduced neutropenia. Clin Pharmacol Ther. 2004 Dec;76:567-578.

30. Jelliffe RW, Schumitzky A, Bayard $\mathrm{D}$, et al. Model-based, goal-oriented, individualised drug therapy - Linkage of population modelling new 'multiple model' dosage design, Bayesian feedback and individualised target goals. Clinical Pharmacokinetics. 1998 Jan;34:57-77.
31. de Jonge ME, Huitema ADR, Schellens JHM, Rodenhuis S, Beijnen JH. Individualised cancer chemotherapy: Strategies and performance of prospective studies on therapeutic drug monitoring with dose adaptation - A review. Clinical Pharmacokinetics. 2005;44:147-173.

32. Hon YY, Evans WE. Making TDM work to optimize cancer chemotherapy: a multidisciplinary team approach. Clinical Chemistry. 1998;44:388-400. 